\title{
AVALIAÇÃO DA CAPACIDADE FUNCIONAL: repensando a assistência ao idoso na Saúde Comunitária
}

\author{
Stefanie Frank ${ }^{1}$ \\ Sílvia Maria Azevedo dos Santos ${ }^{2}$ \\ Andréia Assmann ${ }^{3}$ \\ Karen Lloyd Alves ${ }^{4}$ \\ Nadja Ferreira ${ }^{5}$
}

\section{Resumo}

Esse artigo trata do relato de parte do trabalho de conclusão de curso de graduação em enfermagem de um grupo de acadêmicas da Universidade Federal de Santa Catarina. O objetivo do trabalho foi realizar uma prática assistencial junto ao idoso, ao familiar e ao cuidador numa Unidade Local de Saúde e no âmbito domiciliar, sendo um dos objetivos específicos a avaliação da capacidade funcional de idosos residentes em uma das micro-áreas da área de abrangência da primeira Unidade Local de Saúde (ULS) onde foi desenvolvido o trabalho. A avaliação da capacidade funcional foi realizada com 23 idosos utilizando-se o Older Americans Resources and Services - OARS. No grupo de idosos estudados observamos que o déficit de capacidade mais freqüente foi em relação à limpeza e arrumação da casa, seguido por problemas de controle esfincteriano e controle das finanças. Esses resultados apontam que esses idosos requerem uma assistência voltada para recuperação ou promoção das Atividades da Vida Diária e das Atividades Instrumentais da Vida Diária. Requisitos, esses, que transcendem a linha programática da biomedicina.

Palavras-chave: Capacidade funcional. Idoso. Independência. Enfermagem.

\footnotetext{
Enfermeira pela Universidade Federal de Santa Catarina/UFSC. Professora Substituta do Departamento de Saúde Pública da UFSC. E-mail: stefanie.frank@gmail.com

2 Enfermeira, mestre em Enfermagem pela UFSC, doutora pela UNICAMP, Professora Adjunta do Departamento e do Programa de Pós-Graduação em Enfermagem da UFSC. E-mail: silvia@nfr.ufsc.br

3 Enfermeira pela Universidade Federal de Santa Catarina/UFSC. E-mail: enferufsc@yahoo.com.br

4 Enfermeira pela Universidade Federal de Santa Catarina/UFSC. E-mail: karenlloyda@gmail.com

5 Enfermeira pela Universidade Federal de Santa Catarina/UFSC. E-mail: nadjaferreira@ig.com.br
} 


\section{Introdução}

O crescimento da população de idosos, em números absolutos e relativos, é um fenômeno mundial e está ocorrendo em um nível sem precedentes. Em 1950, eram cerca de 204 milhões de idosos no mundo e, já em 1998, quase cinco décadas depois, este contingente alcançava 579 milhões de pessoas, um crescimento de quase oito milhões de pessoas idosas ao ano (IBGE, 2002). As projeções indicam que, em 2050, a população idosa será de 1.900 milhões de pessoas (ANDREWS, 2000).

No Brasil, segundo o Censo Demográfico de 2000, a proporção de idosos corresponde a $8,6 \%$ da população total ou cerca de quinze milhões de pessoas. Se persistirem as tendências para as taxas de fecundidade e longevidade da população brasileira, no ano 2020 a população idosa poderá exceder 30 milhões de pessoas, chegando a representar quase $13 \%$ do total de habitantes (IBGE, 2002).

Uma das razões para o aumento da população idosa no Brasil é o aumento da expectativa de vida ao nascer. Em 1991, um recém-nascido tinha expectativa de viver 66 anos. Em 2000, essa idade aumentou para 68,6 anos. O ganho, no entanto, não foi igual para homens e mulheres. Enquanto a esperança de vida ao nascer das mulheres aumentou 2,8 anos no período, indo para 72,6 anos, a dos homens teve acréscimo de 2,2 anos, ficando em 64,8 anos. Com isso, aumentou de 7,2 anos para 7,8 anos a diferença na expectativa de vida das mulheres em relação à dos homens. Esta tendência tem sido definida como "feminização da população idosa" (IBGE, 2002). ${ }^{6}$

Com esses fenômenos vimos também surgir problemas antes desconhecidos, isto é, aqueles inerentes ao processo de envelhecimento. Nessa perspectiva, percebendo a necessidade crescente de promoção de mudanças sociais para garantir um envelhecimento digno e com qualidade de vida, é que se buscou pautar o trabalho de conclusão de curso em enfermagem conectado com as demandas dos idosos que foram assistidos pelas acadêmicas.

O presente artigo relata os resultados obtidos na avaliação da capacidade funcional de um grupo de idosos residentes na comunidade em que as acadêmicas desenvolveram seu trabalho ao longo de um se-

${ }^{6}$ Documento eletrônico. 
mestre, utilizando o instrumento Older Americans Resources and Services - OARS.

\section{Revisão de Literatura}

O envelhecimento é um processo de transformação do organismo que ocorre ao longo do ciclo vital de forma universal e progressiva. Hoje, já sabemos que ele é um processo multifatorial que sofre influências intrínsecas e extrínsecas o que faz com que se manifeste de maneira peculiar e individual. Existem diversos estudos e teorias que buscam explicar o envelhecimento em diferentes perspectivas, tais como: as teorias biológicas, psicológicas, teorias do desenvolvimento, teorias sociológicas, entre outras (NERI, 2001; SMELTZER; BARE, 2002).

Como nesse artigo nosso enfoque é a capacidade funcional vamos dar maior destaque às alterações biológicas que possam ser afetadas ao longo do envelhecimento. Assim, compreendemos que as mudanças morfológicas, funcionais e bioquímicas que ocorrem no envelhecimento abrangem todo o organismo (GUIMARÃES et al., 2004), sendo que alguns processos podem ser prevenidos ou eliminados quando a assistência em saúde é efetiva (SMELTZER; BARE, 2002).

Algumas dessas alterações orgânicas são: a perda de força muscular, resistência e agilidade, o que aumenta a probabilidade de ocorrência de doenças crônicas (DUARTE, 1996). Outro aspecto relevante no envelhecimento é a diminuição da capacidade funcional, que acaba por comprometer a realização das atividades da vida diária (AVD) e as atividades instrumentais da vida diária (AIVD). Quando isso ocorre, reflete diretamente sobre o autocuidado fazendo com que, a médio e longo prazo, surja a necessidade de cuidados de longa permanência, geralmente com altos custos (GUIMARÃES et al., 2004; RAMOS, 2003).

O conceito de capacidade funcional abrange a capacidade de o indivíduo manter competência, habilidades físicas e mentais para um viver independente e autônomo. Em outras palavras, capacidade para realização das AVD e AIVD, ou seja, as atividades que fazem parte da rotina do dia-a-dia, como fazer compras, fazer sua contabilidade, manter a continência, banhar-se, deslocar-se e alimentar-se (BRASIL, 1999; GUIMARÃES et al., 2004; ROSA, 2003). São necessários, ainda, pa- 
râmetros multidimensionais para se avaliar a capacidade funcional, tais como: fatores socioeconômicos, culturais, psicossociais, demográficos, estilo de vida adotado, condições de saúde/doença, entre outros (DIOGO, 2003; GUIMARÃES et al., 2004; RICCI; KUBOTA; CORDEIRO, 2005; ROSA, 2003).

A promoção e a manutenção da capacidade funcional em idosos refletem numa melhor qualidade de vida e num envelhecimento bem sucedido, assim como sua recuperação, quando está prejudicada por alguma razão (BRASIL, 1999; OLIVEIRA, 2005). Por outro lado, observa-se que déficits na capacidade de realização das AVD geralmente estão relacionados à fragilidade, institucionalização, dependência de um cuidador e a um maior risco para quedas, morbidade e até a morte (GUIMARÃES et al., 2004; SIQUEIRA et al., 2004).

A avaliação da capacidade funcional vai muito além de um diagnóstico e tratamento de doenças específicas, pois percebe o idoso em seu meio, na sua residência e sua independência em relação aos cuidadores e/ou familiares (BRASIL, 1999; GUIMARÃES et al., 2004; SIQUEIRA et al., 2004).

A capacidade funcional é, atualmente, considerada um novo paradigma de saúde, sendo de valor crucial para prevenção e promoção da saúde do idoso. É através dela que conseguimos diagnosticar, tratar, reabilitar e evitar complicações secundárias das doenças crônico-degenerativas que tão freqüentemente acometem os idosos (DIOGO, 2003; GUIMARÃES et al., 2004; RICCI; KUBOTA; CORDEIRO, 2005; ROSA, 2003;).

Para realizar a avaliação global da capacidade funcional, foram desenvolvidos diversos instrumentos, entre eles o Olders Americans Research and Services (OARS), criado em 1978 pela Duke University Center for the Study of Aging and Human Development, nos EUA. Em 1988, o OARS foi validado no Brasil (BLAY et al., 1988).

\section{Material e Métodos}

As acadêmicas desenvolveram em seu trabalho atividades assistências junto aos usuários idosos de uma Unidade Local de Saúde tanto no âmbito do serviço quanto nos domicílios dessa população, durante o segundo semestre de 2005. Para o planejamento das ações assistenciais 
foi realizado um estudo exploratório acerca da capacidade funcional dos idosos residentes em uma micro-área escolhida por conveniência.

Esta micro-área foi escolhida junto às enfermeiras da Unidade Local de Saúde e os Agentes Comunitários de Saúde em função da segurança, da facilidade de acesso e disponibilidade de um dos agentes em acompanhar as acadêmicas. Ao chegarem aos domicílios elas se apresentavam, explicavam os objetivos de sua visita e solicitavam a permissão do idoso e/ou seu cuidador para aplicação do $O A R S$.

A todos os idosos e/ou seus cuidadores foi solicitada a assinatura do Termo de Consentimento Livre e Esclarecido, conforme preconizado pela Resolução no 196/96 do Conselho Nacional de Saúde e o Código de Ética de Enfermagem. Quando o idoso possuía algum déficit cognitivo ou de memória foi requerido ao cuidador a assinatura do termo de consentimento livre e esclarecido. Vale lembrar, que esse projeto não foi submetido ao Comitê de Ética em Pesquisa com Seres Humanos da Universidade Federal de Santa Catarina porque na ocasião essa não era uma prática corrente para os trabalhos de conclusão de curso.

$\mathrm{O}$ instrumento utilizado nesse trabalho de campo foi o $O A R S$, porque ele permitiu fazer uma avaliação do perfil multidimensional de saúde da população pesquisada. O OARS caracteriza-se como um questionário fechado, que revela os fatores econômicos e sociais, assim como a percepção subjetiva do idoso ou de seu cuidador acerca do seu suporte familiar e sua saúde física e mental (RAMOS, 2003). Ele também avalia a independência do idoso para realização das AVD e as AIVD.

A capacidade funcional foi medida pela dificuldade auto-referida em realizar 14 atividades físicas e instrumentais da vida diária (AVD e AIVD). Vale lembrar, que no instrumento utilizado sete questões são referentes às AVD e as outras sete correspondentes às AIVD. Cada pergunta recebe a pontuação "um" quando o idoso é independente para a atividade questionada, ou "zero" quando ele é dependente para tal atividade. $\mathrm{O}$ resultado final do $O A R S$ corresponde ao somatório da pontuação das 14 atividades avaliadas.

O ponto de corte do OARS obedece aos seguintes critérios: 0 a 3 pontos o idoso é considerado dependente, necessitando de ajuda para realizadas todas ou quase todas as atividades; de 4 a 10 o idoso é considerado parcialmente dependente, ou seja, ele necessita de apoio para 
algumas atividades; já nas pontuações de 11 a 14 o idoso é independente para todas ou praticamente todas as atividades.

Os dados coletados foram agrupados em tabelas, que reuniram as diferentes informações. Com as tabelas em mão, elaboraram-se gráficos para facilitar a análise e proporcionar maior visibilidade aos resultados encontrados.

\section{Resultados e Discussão}

Participaram da pesquisa 23 pessoas com 60 anos ou mais, sendo que a idade média desta amostra foi de 68,9 anos. Dentre os participantes, 16 eram mulheres $(69,5 \%)$ e sete eram homens $(30,5 \%)$. A idade média das mulheres pesquisadas foi de 69,9 anos e dos homens 66,7 anos.

Desses, seis $(16,7 \%)$ não haviam sido cadastrados pelos Agentes Comunitários de Saúde, refletindo o fato de os idosos ainda não serem um marcador de atenção básica, assim como as crianças de 0 a 6 anos, os usuários diabéticos, os hipertensos e as gestantes, entre outros. Um dado preciso sobre os usuários é necessário para que se possa dispor de informações atualizadas sobre o perfil da população. Somente assim é possível fazer-se um planejamento e implementar-se uma assistência de qualidade, isto é, que vá ao encontro das demandas da população assistida.

Com relação à capacidade funcional, o déficit mais freqüentemente encontrado na amostra pesquisada foi em relação à limpeza e arrumação da casa, tanto para as mulheres quanto para os homens. Alguns idosos referiram a necessidade de auxílio para realizar as atividades que exigem maior força muscular, como passar panos no chão e limpar janelas. Outros relataram não fazer estas atividades de modo algum, devido às suas limitações físicas ou por não saber como realizá-las.

A maioria dos idosos referiu-se independente para a maior parte das atividades, principalmente para as AVD. Em relação à dependência relativa, observou-se que uma pequena parcela dos idosos possui maior dependência em relação às AIVD. Apenas um idoso apresentou dependência completa, conforme pode ser visto no gráfico 1 . 


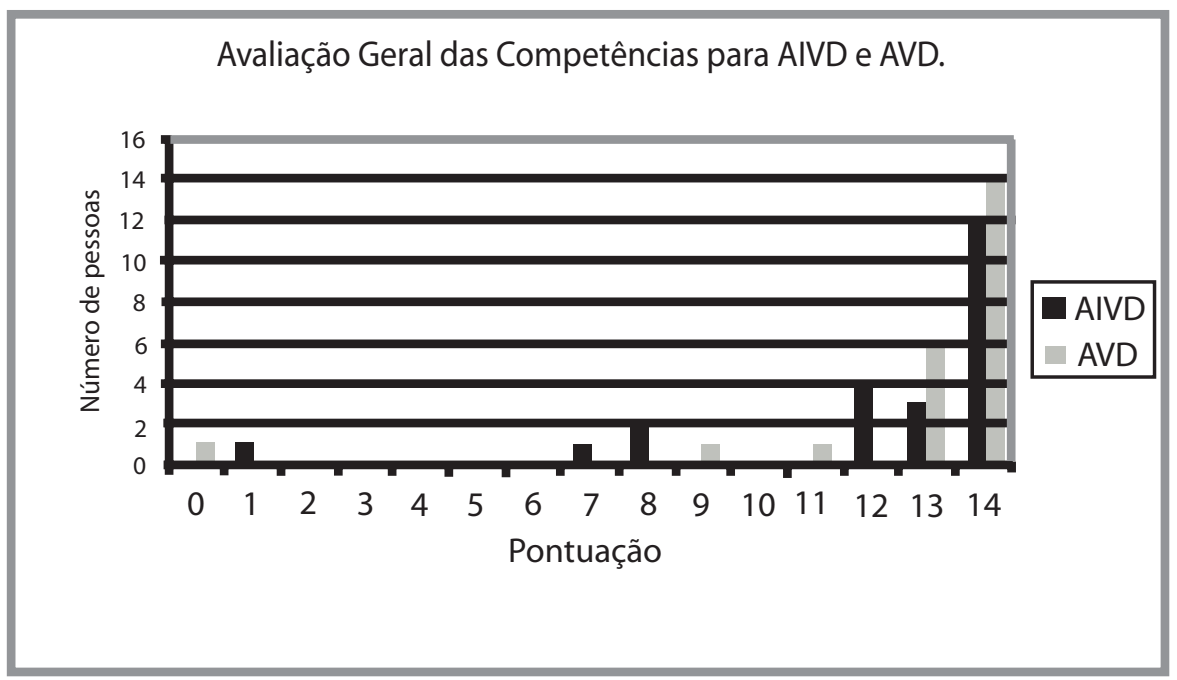

GRÁFICO 1: Avaliação Geral das Competências para AIVD e AVD nos idosos da Micro-área mapeada, em outubro de 2005.

Na seqüência os déficits de maior prevalência foram os controles esfincterianos e o controle das finanças. As atividades com menor déficit referido foram cuidados com a aparência e capacidade para alimentar-se.

Observou-se que a pontuação geral é mais elevada na faixa etária de 65 a 70 anos, refletindo uma maior independência desses idosos, como pode ser observado no gráfico 2 .

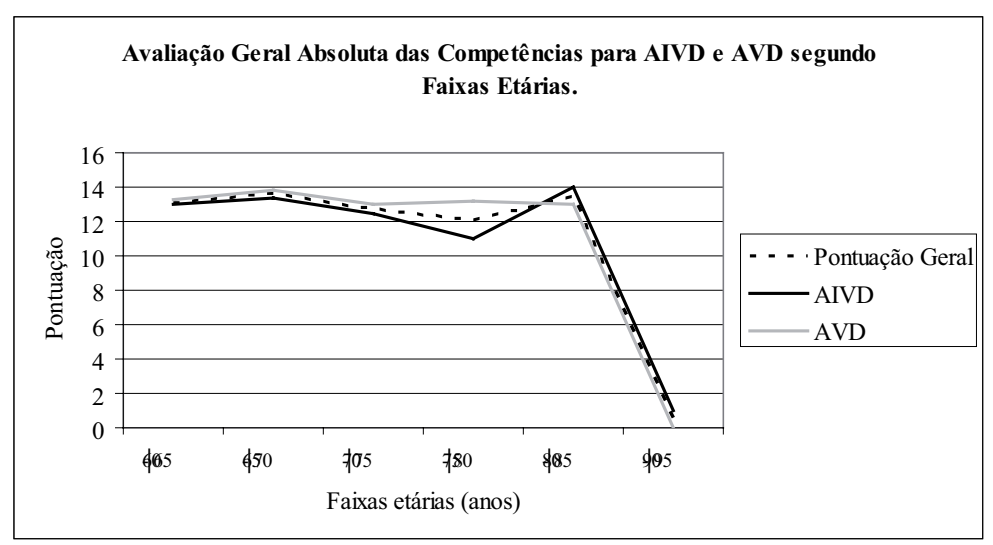

GRÁFICO 2: Avaliação Geral Absoluta das Competências para AIVD e AVD segundo Faixas Etárias dos idosos da Micro-área mapeada, em outubro de 2005. 
Nas atividades de limpeza e arrumação da casa, observou-se que aproximadamente $40 \%$ dos idosos necessitavam de auxílio para realizá1a. Nenhum dos idosos avaliados precisava de veículos especiais, como ambulâncias, para seu transporte. A faixa etária de 85 anos a 90 anos foi suprimida por não haver nenhum idoso com essa idade.

Pode-se atentar no Gráfico 3 que aproximadamente 80\% dos idosos até 75 anos apresentaram plena capacidade para realizar as AVD e AIVD sem auxílio. Identificou-se um decréscimo abrupto da capacidade para independência na faixa etária de 90-95 anos, refletindo uma maior necessidade de apoio de familiares e/ou cuidadores para a realização das atividades diárias.

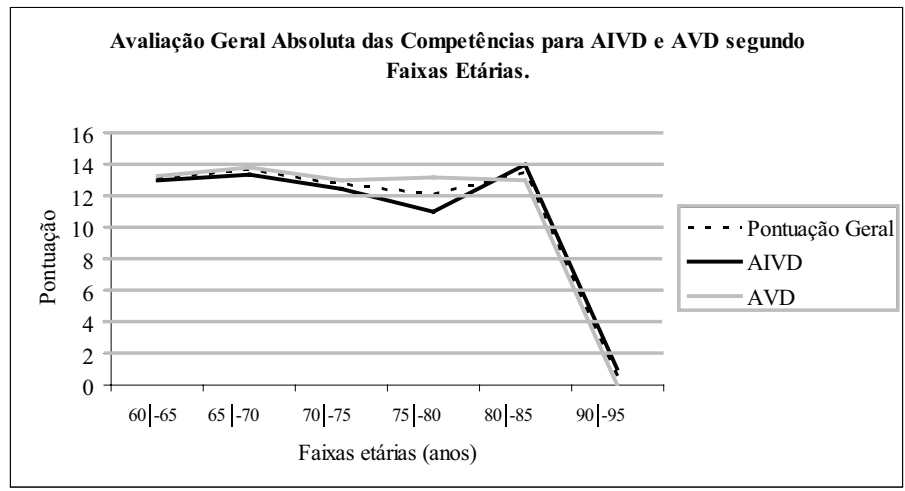

GRÁFICO 3: Avaliação Geral das Competências para AIVD e AVD segundo a Faixa Etária da população idosa na Micro-área mapeada, em Números Relativos e de Acordo com a Autonomia, em outubro de 2005.

De modo geral, os idosos avaliados apresentaram dependência relativa para arrumar e limpar a casa, com aproximadamente $37 \%$, seguida do controle das finanças e problemas no controle esfincteriano, ambos com aproximadamente $31 \%$. Não foi encontrada dependência relativa nas capacidades para alimentar-se e cuidar da aparência.

\section{Conclusões}

Avaliando os resultados obtidos, concluiu-se que grande parte dos idosos assistidos estava com sua capacidade funcional preservada para o desenvolvimento das atividades da vida diária e atividades funcionais da vida diária, revelando autonomia e independência.

Acredita-se que identificar a capacidade funcional do idoso e 
sua competência para realizar as atividades da vida diária aproxima o profissional de saúde da realidade vivida pelos mesmos e seus cuidadores. Também possibilita um melhor planejamento da assistência a ser prestada especialmente quando há déficits, porque irão interferir na qualidade de vida do idoso e de seu familiar cuidador.

No que diz respeito à enfermagem pensamos que ela deva estar familiarizada com os acontecimentos e a realidade vivida pelos usuários, para assim levá-los à reflexão sobre a situação. Também é importante incentivar e apoiar os idosos em suas ações de autocuidado, visando prevenir o surgimento de déficits e/ou reabilitando quando necessário.

A utilização de uma escala como o $O A R S$ possibilitou a classificação dos idosos num paradigma diferente daquele da biomedicina, que tende a colocar o diagnóstico médico à frente do ser humano ao qual se assiste. Ao utilizarmos essa escala tivemos apenas uma dificuldade que foi quanto à forma de pontuar. O ponto de corte deste instrumento varia de 1 a 14, contudo, obteve-se a pontuação zero numa das entrevistas quando um dos idosos pesquisados apresentou dependência total para todas as atividades questionadas.

Espera-se que este trabalho sirva como estímulo para acadêmicos, profissionais da área da saúde e docentes a desenvolverem novos estudos que permitam mapear a real situação da capacidade funcional dos idosos residentes na comunidade. Somente de posse de informações precisas pode-se compreender melhor o ser idoso, suas demandas e as de seus familiares e/ou cuidadores.

\title{
EVALUATION OF THE FUNCTIONAL CAPACITY: rethinking the assistance to the aged one in the Communitarian Health
}

\begin{abstract}
This Article treats about a part of the course conclusion work of a group of nursing students at the Federal University of Santa Catarina. The objective of this work was realize an assistance with older ones, the family and the carers at one Public Health Station and in their homes, being one of the specific objectives the evaluation of the func-
\end{abstract}


tional capacity of ages residents in a area of the health station where the work was realized. The evaluation of the functional capacity was realized with 23 ages using the Older Americans Resources and Services - OARS. In this aged group, the deficit of more frequency was in relation to the cleanness and arrangement of the house, followed by problems of sphincter control and finances control. These results appoint that the aged one requests a assistance that turns to the recuperation ore promotion of the Daily Live Activities and the Daily Live Instrumental Activities. There are requisitions that transcend the programmatically line of the biomedicine.

Keywords: Functional capacity. Age one. Independence. Nursing.

\section{Referências}

ANDREWS, G. A. Los desafíos del processo de envejecimiento em las sociedades de hoy y del futuro. In: ENCUENTRO LATINOAMERICANO Y CARIBENHO SOBRE LAS PERSONAS DE EDAD, 1999, Santiago. Anais ... Santiago: CELADE, 2000. P. 247-256.

BLAY, Sergio ; RAMOS, L. R. ; MARI, Jair Jesus . Validity of a Brazilian Version of the Older Americans Resources and Services (OARS) Mental Health Screening Questionnaire. Journal of the American Geriatric Society, Ames, v. 36, p. 687-692, 1988.

BRASIL. Ministério da Saúde. Portaria no 1395, de 10 de dez. 1999. Aprova a Política Nacional do Idoso. Disponível em: < http://www.cremesp.org. br/library/modulos/legislacao/versao_impressao.php?id=6621>. Acesso em: 26 ago. 2007.

DIOGO, M. J. D. Satisfação Global com a Vida e Determinados Domínios entre Idosos com Amputação de Membros Inferiores. Revista Panamericana de Salud Pública, Washington, v.13, n. 6, jun. 2003. Disponível em: $<$ http:// www.scielosp.org/scielo.php?pid=S1020-49892003000500008\&script $=$ sci_arttext\&tlng=pt>. Acesso em: 26 jul. 2006.

DUARTE, Y. A. O. Princípios de Assistência de Enfermagem Gerontológica. In: NETTO, M. P. Gerontologia, São Paulo: Atheneu, 1996. P. 222 - 229. 
GUIMARÃES, L. H. C. T.; GALDINO, D. C. A.; MARTINS, F. L. M.; ABREU, S. R.; LIMA, M.; VITORINO, D. F. M. Avaliação da Capacidade Funcional de Idosos em Tratamento Fisioterapêutico. Revista Neurociências, São Paulo, v. 12, n. 3, jul./set. 2004. Disponível em: < http://www.unifesp.br/dneuro/neuro ciencias/vol12_3/capacid_funcional.htm>. Acesso em: 25 jul. 2006.

IBGE. Perfil dos Idosos Responsáveis pelos Domicílios no Brasil: 2000. Estudos e Pesquisas: informação demográfica e socioeconômica: IBGE, Rio de Janeiro, n. 9, 2002. Disponível em: <http://www.ibge.gov.br/home/estatistica/populacao/perfilidoso/perfidosos2000.pdf.> Acesso em: 28 jun. 2005.

NERI, A. L. Palavras-Chave em Gerontologia. Campinas: Alínea, 2001.

OLIVEIRA, B. H. D. Sobre a Saúde e Qualidade de Vida no Envelhecimento. Textos Envelhecimento, Rio de Janeiro, v. 8, n. 1, 2005. Disponível em:

$<$ http://www.unati.uerj.br/tse/scielo.php?script=sci_arttext\&pid $=$ S151759282005000100008\&lng=pt\&nrm=iso>. Acesso em: 25 jul. 2006.

RAMOS, L. R. Fatores Determinantes do Envelhecimento Saudável em Idosos Residentes em Centro Urbano: projeto epidoso, São Paulo. Caderno de Saúde Pública, Rio de Janeiro, v. 19, n. 3, jun. 2003. Disponível em:

$<$ http://www.scielo.br/pdf/csp/v19n3/15882.pdf >. Acesso em 22 jul. 2007.

RICCI, N. A.; KUBOTA, M. T.; CORDEIRO, R. C. Concordância de observações sobre a capacidade funcional de idosos em assistência domiciliar. Revista de Saúde Pública, São Paulo, v. 39, n. 4, 2005. Disponível em:

$<$ http://www.scielo.br/pdf/rsp/v39n4/25540.pdf $>$. Acesso em: 25 jul. 2006.

ROSA, T. E. C.; BENÍCIO, M. H. D.; LATORRE, M. R. D. O.; RAMOS, L. R. Fatores Determinantes da Capacidade Funcional entre Idosos. Revista de Saúde Pública, São Paulo, v. 37, n. 1, fev. 2003. Disponível em: <http://www.scielo. br/scielo.php?pid=S0034-89102003000100008\&script $=$ sci_arttext\&tlng=pt $>$. Acesso em: 25 jul. 2006.

SIQUEIRA, A. B.; CORDEIRO, R. C.; PERRACINI, M. R.; RAMOS, L. R. Impacto Funcional da Internação Hospitalar de Pacientes Idosos. Revista de 
Saúde Pública, São Paulo, v. 38, n. 5, out. 2004. Disponível em:

$<$ http:// www.scielo.br/scielo.php?script=sci_arttext\&pid=S0034$89102004000500011 \&$ lng=pt\&nrm=iso\&tlng=pt $>$. Acesso em: 23 ago. 2006.

SMELTZER, S. C.; BARE, B. G. Brunner \& Suddarth: tratado de enfermagem médico-cirúrgica. 9. ed. Rio de Janeiro: Guanabara Koogan, 2002.

Recebido em: 11.12.2006

$1^{a}$ revisão: 21.05 .2007

Aceite final: 31.05 .2007 